\title{
Cardiovascular comorbidities among patients with metastatic colorectal cancer
}

\author{
JA Overbeek ${ }^{1}$, Z Zhao*2, MPP van Herk-Sukel ${ }^{3}$, BL Barber ${ }^{4}$ and RMC Herings ${ }^{5}$
}

Article Base : Oral presentation made at the 2011 European Multidisciplinary Cancer Congress (ECCO 16th, ESMO 36th and ESTRO 30th Annual Meeting).

\begin{abstract}
Aim: As comorbidities may impact treatment decisions, prognoses and quality of care, this study determined the rate of comorbid cardiovascular diseases in patients with metastatic colorectal cancer $(\mathrm{mCRC})$.

Methods: From the PHARMO Record Linkage System in The Netherlands, all patients with a hospital discharge code for CRC and distant metastasis from 2000-2008 were selected. Prevalent cardiovascular comorbidities were assessed during the 12 months prior to the index date (the first discharge diagnosis defining metastases). Cardiovascular comorbidities were captured using cardiovascular drug use and hospital admission data. 2964 patients with mCRC were included in the analysis. Mean ( \pm standard deviation) age at diagnosis was $68( \pm 12)$ years and $53 \%$ were male.

Results: Cardiovascular comorbidities were observed in $52 \%$ of patients. Of patients identified by drug use, the most commonly used agents were antithrombotic agents (54\%), beta-blocking agents (46\%), and agents acting on the renin-angiotensin system (45\%). Of patients hospitalised for cardiovascular comorbidities, about one-third were hospitalised for cardiac dysrhythmia (39\%), followed by congestive heart failure (19\%) and hypertension (18\%).

Conclusions: Cardiovascular comorbidities are common in patients with $\mathrm{mCRC}$, which is likely to be explained by the high mean age at diagnosis. Consideration of these conditions should be integral to the treatment strategy in individual patients with $\mathrm{mCRC}$.
\end{abstract}

Keywords: cardiovascular disease, comorbidity, metastatic colorectal cancer

\section{Introduction}

Colorectal cancer (CRC) is one of the most common malignancies, third after lung and prostate cancer in men and second only to breast cancer in women [1]. In a significant proportion of patients with $\mathrm{CRC}$, metastases are already present at diagnosis and many patients diagnosed at earlier stages will also eventually develop metastatic disease [2].

Current pharmacological treatment options for patients with metastatic CRC ( $m C R C$ ) include the use of chemotherapy in combination with monoclonal antibodies targeting vascular endothelial growth factor (VEGF) or the epidermal growth factor receptor (EGFR) (in patients with wild-type KRAS tumours). These treatments have led to significant improvements in the treatment outcomes of $\mathrm{mCRC}$ patients in recent years [3]. With

\footnotetext{
* Correspondence: zhongyun@amgen.com

${ }^{2}$ Amgen Inc, One Amgen Center Drive, Thousand Oaks, CA 91320-1799, USA.
}

the incidence rates of $\mathrm{mCRC}$ increasing and the treatment outcomes improving, the number of prevalent $\mathrm{mCRC}$ cases keeps increasing [4]. Therefore, many more patients with $\mathrm{mCRC}$ will be undergoing treatment, and cope with this progressive disease for a longer period of time. Many of these patients will have concomitant conditions which have various clinical implications. Comorbidities, defined as medical conditions exist simultaneously but independently with the primary medical condition in a patient, often necessitate modifications of and/or adversely influence the chosen therapy; concomitant diseases are independent determinants of prognosis (regardless of the chosen therapy) and their presence is also an indicator of the complexity of the clinical needs of each patient [5]. Evaluation of the presence of cardiovascular comorbidities is particularly important in the $\mathrm{mCRC}$ population as some of the newer treatments are associated with adverse events related to the cardiovascular system [6,7]. These adverse events include, among others, thromboembolism, hypertension, haemorrhage

(C) 2012 Zhao et al; licensee Herbert Publications Ltd. This is an open access article distributed under the terms of Creative Commons Attribution License (http://creativecommons.org/licensesby/2.0), This permits unrestricted use, distribution, and reproduction in any medium, provided the original work is properly cited 
and cardiotoxicity, and may be even more dramatic when concomitant cardiovascular disease is present.

Data regarding the prevalence of cardiovascular comorbidities in patients with $\mathrm{MCRC}$ are, however, limited. This study, therefore, assessed the prevalence of pre-existing cardiovascular comorbidities (existence of cardiovascular diseases with $\mathrm{mCRC}$ at the same time) among a populationbased cohort of patients with $\mathrm{mCRC}$ in The Netherlands.

\section{Materials and methods Data source}

Data were obtained from the PHARMO medical record linkage system (PHARMO RLS), which consists of multiple observational databases linked on a patient level, covering 3.2 million inhabitants of geographic defined areas in The Netherlands. Databases relevant for this study include the Dutch National Medical Register (LMR) and the community pharmacy database (outpatient). The hospital records contain detailed information concerning admissions for more than 24 hours and admissions for less than 24 hours for which a bed is required, including primary and secondary diagnoses, procedures and dates of hospital admission and discharge [8]. All diagnoses are coded according to the International Classification of Diseases, Ninth Revision, Clinical Modification (ICD-9-CM). The drug dispensing histories from outpatient pharmacies contain data on the dispensed drug, prescriber, dispensing date, amount dispensed, prescribed dose regimens and thus the duration of use. All drugs are coded according to the Anatomical Therapeutic Chemical (ATC) Classification.

\section{Patient population}

Patients aged $\geq 18$ years diagnosed with $\mathrm{mCRC}$ were identified by hospitalisation codes between 1 January 2000 and 31 December 2008. All patients with a primary or secondary hospital discharge diagnosis for CRC (ICD-9-CM code 153.x (excluding 153.5), 154.0, 154.1, and 154.8) and metastasis (ICD-9CM code 196.0, 196.1, 196.3, 196.5, 197.x (excluding 197.5), 198.x, and 199.0) were selected $[9,10]$.

To be included in the study, the hospital admission date for distant metastasis needed to occur no more than 30 days before the hospital admission date for CRC or anywhere after the hospital admission date for CRC, on condition that the patient was not hospitalised for cancer other than CRC. The first metastasis discharge diagnosis date served as the index date. Patients with metastasis diagnosis within 10 years before the index date were excluded as these metastases might be related to a primary tumour other than $\mathrm{CRC}$. To be able to define comorbidities in recent history based on drug use as well as hospitalisations, patients needed to have at least 12 months of history in the PHARMO RLS.

\section{Outcome measures}

For all study patients the following characteristics were determined at index date: gender, age, site of primary tumour (colon or rectal), site of metastasis and available history in PHARMO RLS. Prevalent comorbidities were assessed during the first year prior to the index date. Cardiovascular comorbidities were identified using drug dispense information in the outpatient setting and discharge diagnoses in the hospital admission database. Information on drugs dispensed in the outpatient setting included: antihypertensives (ATC code C02), diuretics (ATC code C03 (excluding C03C), beta-blocking agents (ATC code C07), calcium-channel blockers (ATC code C08), agents acting on the renin-angiotensin system (ATC code C09 (excluding C09X), antithrombotic agents (ATC code B01), and cardiac therapy (ATC code C01). In the hospital admission database, cardiovascular comorbidities were determined based on primary and secondary hospital discharge diagnoses, including: hypertension (ICD-9-CM code 401-405), coronary artery disease (ICD-9-CM code 414.0), cardiac dysrhythmia (ICD9-CM code 427), congestive heart failure (ICD-9-CM code 402.01, 402.11, 402.91 and 428), acute myocardial infarction (ICD-9-CM code 410), other ischaemic heart disease (ICD-9-CM code 411, 413 and 414 (excluding 414.0), venous thromboembolism (ICD9-CM code 451.1, 451.2, 451.81, 451.9, 453.2, 453.8 and 453.9), arterial thromboembolism (ICD-9-CM code 444), and stroke (ICD-9-CM code 362.3, 433.1, 434.1, 435 and 436).

\section{Statistical analysis}

Patient characteristics and cardiovascular comorbidities prior to $\mathrm{mCRC}$ diagnosis were presented descriptively by the number and proportion of patients receiving these agents or being hospitalised for these diagnoses, with 95\% confidence intervals. All data were analysed using SAS programs organised within SAS $^{\circledR}$ Enterprise Guide version 4.0 (SAS Institute Inc, Cary, NC, USA) and conducted under UNIX using SAS version 9.2.

\section{Results}

Overall, 2964 patients with $\mathrm{mCRC}$ were included in the analyses. The study population included similar numbers of males and females (Table 1) and patients aged 70-79 represented the largest group by age (32\%); mean ( \pm SD) age was 68 ( \pm 12 ) years. Colon cancer was more common than rectal cancer as primary tumour (69\% and $31 \%$, respectively). The most common location of metastases was the liver ( $43 \%$ in patients with colon cancer and $42 \%$ in patients with rectal cancer as primary tumour).

The prevalence of cardiovascular comorbidities, as identified by drug use and/or hospitalisations, in the year prior to index date is shown in (Table 2). Overall, cardiovascular comorbidities were observed in $52 \%$ of patients. The majority of these patients were identified by drug use (96\%) and only a small proportion (19\%) was identified by hospitalisations of all patients identified by drug use, the most commonly used agents were antithrombotic agents (54\%), followed by beta-blocking agents (46\%), and agents acting on the renin-angiotensin system (45\%) of the patients hospitalised for cardiovascular comorbidities in the year prior to index date, about one-third was hospitalised for 
Table 1. Characteristics of patients hospitalised for mCRC

Total

$\mathbf{N}=\mathbf{2 9 6 4} n$ (\%)

\section{Gender}

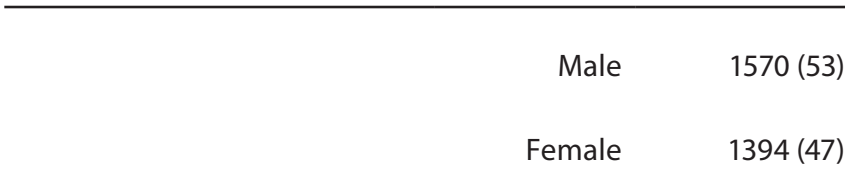

\section{Age (years)}

$\begin{array}{rr}\geq 18-49 & 188(6) \\ 50-59 & 471(16) \\ 60-69 & 810(27) \\ 70-79 & 961(32) \\ \geq 80 & 534(18) \\ \text { mean } \pm \text { SD } & 68 \pm 12\end{array}$

mCRC diagnosis

Malignant neoplasm of

$2032(69)$

colon

Liver metastases
$\begin{aligned} & \text { Metastases of the retroperit } \\ & \text { neum or peritoneum }\end{aligned}$
Lung metastases
Malignant neoplasm of
rectum

$876(43)$

$434(21)$

Liver metastases

$172(9)$

$932(31)$

$392(42)$

Metastases of the retroperito-

90 (10)

neum or peritoneum

Lung metastases

$123(13)$

Metastases at other sites

$327(16)$

mCRC diagnosis period
Table 2. Existing cardiovascular comorbidities in patients hospitalised for $\mathrm{MCRC}$

Total

$\mathbf{N}=\mathbf{2 9 6 4} \mathbf{n}(\%)$

Drug use and/or hospitalisation for

$1544(52)$ cardiovascular comorbidities

Drug use for cardiovascular comorbidi-

1479 (96)

ties

Antihypertensives

$33(2)$

Diuretics

Beta-blocking agents

$683(46)$

Calcium-channel blockers

$306(21)$

Agents acting on the renin-angiotensin

$665(45)$

system

Antithrombotic agents

804 (54)

Cardiac therapy

Hospitalisation for cardiovascular

297 (19)

comorbidities

Hypertension

$52(18)$

Coronary artery disease

Cardiac dysrhythmia

$116(39)$

Congestive heart failure

55 (19)

Acute myocardial infarction

Other ischaemic heart disease

$43(14)$

Venous thromboembolism

$31(10)$

Arterial thromboembolism

Stroke

mCRC, metastatic colorectal cancer

$\begin{array}{ll}2000-2004 & 1633(55) \\ 2005-2008 & 1331(45)\end{array}$

History in PHARMO RLS (mean \pm SD [in years]) $6 \pm 3$ mCRC, metastatic colorectal cancer; RLS, Record Linkage System; SD, standard deviation 
cardiac dysrhythmia (39\%), followed by congestive heart failure (19\%) and hypertension (18\%).

(Figure 1) shows the rate of cardiovascular comorbidities per age category for males and females separately. For both males and females, the proportion of patients with cardiovascular comorbidities increased with increasing age.

\section{Discussion}

To our knowledge, there are limited data on the prevalence of cardiovascular comorbidities in patients with $\mathrm{mCRC}$. In this retrospective cohort study the prevalence of cardiovascular comorbidities among patients with $\mathrm{mCRC}$ was determined using both cardiovascular drug use and hospital admission data. Our analyses illustrate that cardiovascular comorbid conditions were observed in more than half of patients in the year prior to the first $\mathrm{mCRC}$ hospitalisation. Most patients used antithrombotic agents, followed by beta-blocking agents and agents acting on the renin-angiotensin system. This high prevalence might be anticipated given that the majority of patients were $>65$ of age.

The rates of cardiovascular comorbidities that were observed in this study can be compared with another study evaluating the extent of cardiovascular comorbidities identified on the basis of ICD-9 diagnosis codes in patients with mCRC [11]. Using this approach, pre-existing cardiovascular diseases were observed in $62 \%$ of 12,648 newly diagnosed patients with $\mathrm{mCRC}$ in the USA. Our results using pharmacy and hospitalisation data are therefore broadly similar to those from the US study using diagnostic criteria. It should be noted, however, that our results reflect the population in The Netherlands and might not be applicable to $\mathrm{MCRC}$ populations in other countries. A previous Dutch study on newly diagnosed patients with CRC in the period 1995-1999 showed that about $32 \%$ of the patients suffered from cardiovascular disease and $26 \%$ suffered from hypertension [12]. This would result in a prevalence of $58 \%$ maximally, as more than one condition per patient was possible, which is comparable to our prevalence estimate.

Our results were generated using outpatient drug dispensing data and hospitalisation discharge diagnoses that are related to cardiovascular diseases as a proxy for the presence of cardiovascular comorbid conditions. Hospital admission data were used to select the cardiovascular morbidities that led to hospitalisation. Pharmacy data were used to select the cardiovascular conditions that were treated in outpatient setting and to provide a more complete list of actively treated conditions [13]. However, algorithms for identifying comorbidities based on pharmacy drug use have been reported to be more specific but less sensitive than algorithms based on diagnosis criteria [14]. This may reflect the fact that this approach will only identify patients who receive pharmacological treatment for their disease. It is also worth to note that some patients might have received adjuvant therapies before metastasis diagnosis and some of adjuvant therapies, such as 5-fluorouracil therapy, could cause cardiotoxicity [15]. However, use of adjuvant therapy was not included in the current analysis.

\section{Conclusions}

In conclusion, cardiovascular comorbidities are common in patients with $\mathrm{mCRC}$, which is likely to be explained by the high mean age at diagnosis. Consideration of these comorbid conditions should be an integral part of the treatment strategy in individual patients with $\mathrm{mCRC}$.

\section{Article History}

Editor: David J. MacEwan, University of East Anglia, UK. EIC: G.J. Peters, VU University Medical Center, Netherlands. Recieved: 21-Dec-2011;

Accepted: 13-Jan-2012 Published : 14-Feb-2012

Figure 1

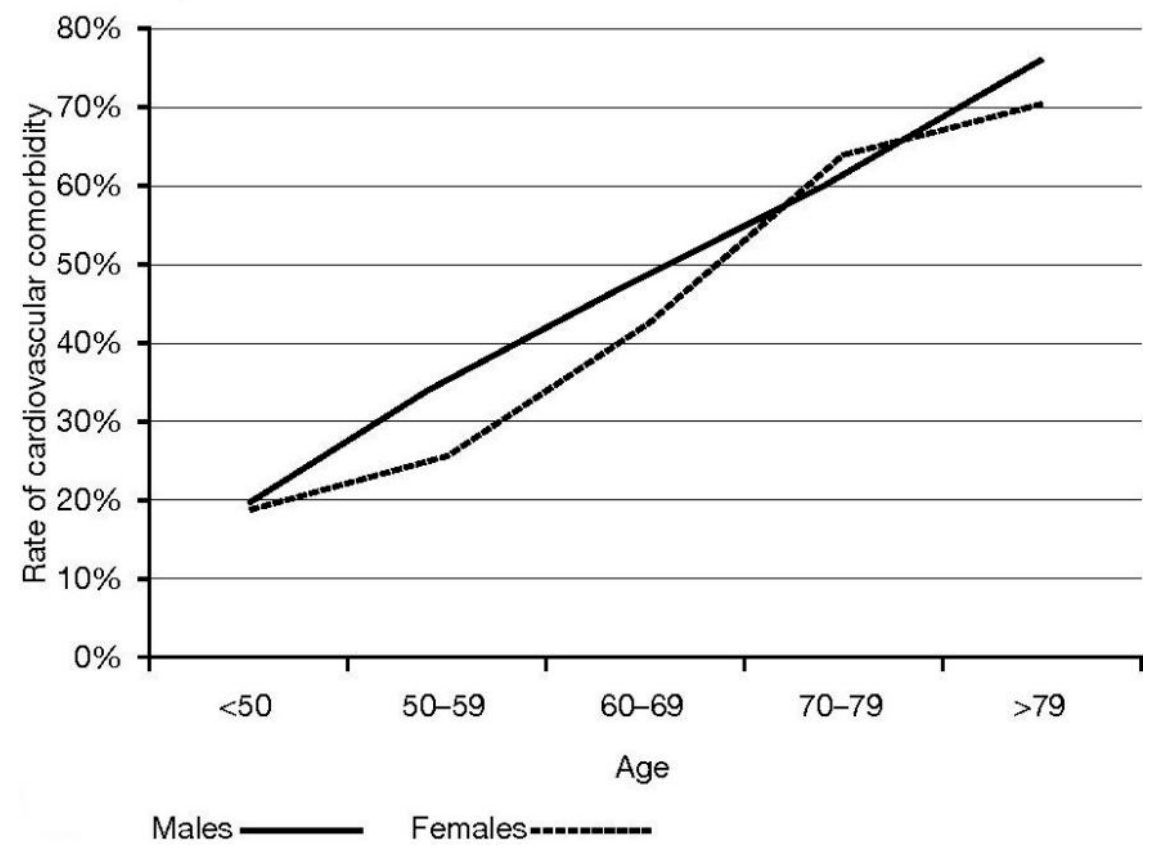




\section{Author contributions}

JA Overbeek, Z Zhao, B Barber, MPP van Herk-Sukel and RMC Herings conceived the study, planned its design and its coordination, and drafted the manuscript. MPP van Herk-Sukel performed the statistical analyses. All authors read and approved the final the manuscript.

\section{Conflict of interest}

All authors had full access to the data and had final responsibility for the decision to submit the manuscript. JA Overbeek, MPP van Herk-Sukel and RMC Herings are employees of the PHARMO Institute for Drug Outcomes Research which received funding from Amgen Inc in connection with the conduct of this study. Z Zhao and BL Barber are employees of Amgen Inc.

\section{Funding}

This study was financially supported by Amgen Inc. No limitations were set with regard to the conduct of the study and the writing of the manuscript.

\section{Acknowledgements}

Editorial assistance was provided by ApotheCom ScopeMedical Ltd, funded by Amgen Inc.

\section{Author information}

'PHARMO Institute for Drug Outcomes Research, PO Box 85222, 3508 AE Utrecht, The Netherlands.

${ }^{3}$ PHARMO Institute for Drug Outcomes Research, PO Box 85222, 3508 AE Utrecht, The Netherlands.

${ }^{4}$ Amgen Inc, One Amgen Center Drive, Thousand Oaks, CA 91320-1799, USA.

${ }^{5}$ PHARMO Institute for Drug Outcomes Research, PO Box 85222, 3508 AE Utrecht, The Netherlands; Department of Health Policy \& Management, Erasmus Medical Centre, 3015 CE Rotterdam, The Netherlands.

\section{References}

1. Vrdoljak E, et al. Phase II study of bevacizumab in combination with capecitabine as first-line treatment in elderly patients with metastatic colorectal cancer. Anticancer Drugs 22, 191-197.

2. Ramsey, S. D. et al. Lifetime cancer-attributable cost of care for long term survivors of colorectal cancer. Am J Gastroenterol 97, 440-445.

3. Van Cutsem E, et al. ESMO Guidelines Working Group (2010) Advanced colorectal cancer: ESMO Clinical Practice Guidelines for treatment. Ann Oncol 21, v93-v97.

4. Lemmens, V. E. et al. Co-morbidity leads to altered treatment and worse survival of elderly patients with colorectal cancer. Br J Surg 92, 615-623.

5. Coebergh JW, et al. Serious co-morbidity among unselected cancer patients newly diagnosed in the southeastern part of The Netherlands in 1993-1996. J Clin Epidemiol 52, 1131-1136.
6. Chaudhary $P$, cardiovascular effects of EGFR (epidermal growth factor receptor) monoclonal antibodies. Cardiovasc Hematol Agents Med Chem 8: 156-163.

7. Vaklavas C, et al. Anti-vascular endothelial growth factor therapies and cardiovascular toxicity: what are the important clinical markers to target? Oncologist 15, 130-141.

8. Dutch Hospital Data. Available at: http://www. dutchhospitaldata.nl (accessed March 2011).

9. Song, $X$. et al. Cost of illness in patients with metastatic colorectal cancer. J Med Econ 14, 1-9.

10. Song $X$, et al. Treatment patterns and metastasectomy among $\mathrm{mCRC}$ patients receiving chemotherapy and biologics. Curr Med Res Opin 27, 123-130.

11. Fu AZ, et al. Comorbidities in patients with metastatic colorectal cancer (mCRC). Eur J Cancer Suppl 7, 339.

12. Lemmens $V E$, et al. Which comorbid conditions predict complications after surgery for colorectal cancer? World J Surg 31, 192-199.

13. Sloan, K. L. et al. Construction and characteristics of the RxRisk-V: a VA-adapted pharmacy-based case-mix instrument. Med Care 41, 761-774.

14. Rector TS, et al. Specificity and sensitivity of claims-based algorithms for identifying members of Medicare+Choice health plans that have chronic medical conditions. Health Serv Res 39, 1839-1857.

15. Gradishar, W. J. et al. 5-Fluorouracil cardiotoxicity: a critical review. Ann Oncol 1, 409-414. 